\title{
Nivel de pobreza y estado nutricional asociados a parasitosis intestinal en estudiantes, Huánuco, Perú, 2010
}

\author{
Poverty and nutritional status-related intestinal parasitism in students, Huanuco,
}

Peru, 2010

\author{
César Gabriel Berto Moreano ${ }^{1,2}$, Judith Cahuana Aparco ${ }^{1,2}$, Jesús Kevin Cárdenas \\ Gallegos $^{1,2}$, Nataly Ruth Botiquín Ortiz', Claudia Angélica Balbín Navarro ${ }^{1,3}$, Paul \\ Jesús Tejada Llacsa ${ }^{1,2}$, Esteffany Jennifer Calongos Porras ${ }^{1}$ \\ ${ }^{1}$ Universidad Nacional Mayor de San Marcos. Quinto año de Medicina Humana. \\ ${ }^{2}$ Asociación para el Desarrollo de la Investigación Estudiantil en Ciencias de la Salud.
}

${ }^{3}$ Sociedad Científica San Fernando.

\begin{abstract}
Resumen
Introducción: Se ha encontrado una prevalencia alta de parasitosis en escolares de la amazonía; pese a ello, no hay estudios realizados que revisen su asociación con el nivel socioeconómico y estado nutricional. Objetivos: Determinar la relación entre nivel de pobreza y estado nutricional con la presencia de parásitos intestinales en estudiantes escolares del caserío Venenillo, Huánuco. Diseño: Estudio transversal-analítico. Lugar: Caserío de Venenillo, Huánuco. Participantes: Estudiantes escolares de la única institución educativa del centro poblado. Intervenciones: A 42 estudiantes se les realizó examen coproparasitológico mediante observación directa con lugol y técnica de sedimentación rápida de Lumbreras. El nivel de pobreza fue determinado mediante el índice de necesidades básicas insatisfechas (NBI); y el grado de desnutrición, por medio del índice de Waterlow. El análisis estadístico fue realizado mediante el coeficiente gamma de Goodman y Kruskal. Principales medidas de resultados: Relación de parasitosis intestinal con pobreza y estado nutricional. Resultados: Los parásitos intestinales estuvieron presentes en 97,6\%. Se encontró asociación significativa entre el nivel de parasitismo y el nivel de pobreza $(p=0,02 ;$ gamma $=0,82)$. No se encontró asociación significativa entre nivel de desnutrición y parasitismo $(p=0,77)$. Conclusiones: La parasitosis intestinal se asoció al nivel de pobreza, no así con el nivel de desnutrición en la población de estudio.
\end{abstract}

Palabras clave: Parasitosis intestinales, estado nutricional, estudiantes, pobreza, Perú.

\begin{abstract}
Introduction: Studies have reported high prevalence of parasitic disease in students from the jungle although none has associated it with poverty and nutritional factors. Objectives: To determine the relationship between poverty and nutritional factors with the presence of intestinal parasites in students from a Huanuco village. Design: Cross-sectional study. Setting: Venenillo hamlet, Huanuco, Peru. Participants: Students from the only school in Venenillo. Interventions: Parasitological examination by Lugol's iodine direct examination and Lumbreras' fast sedimentation technique was performed in 42 students. Level of poverty was determined by the unsatisfied basic needs index and degree of malnutrition by the Waterlow index. Statistical analysis was performed using Goodman and Kruskal's gamma coefficient. Main outcome measures: Intestinal parasitosis association with poverty and nutritional status. Results: Presence of intestinal parasites was $97.6 \%$. A strong association was found between parasitism and level of poverty ( $p=0.02$, gamma=0.82). There was no significant association between level of malnutrition and parasitism. Conclusions: Association between intestinal parasitosis and poverty was encountered in the studied population.
\end{abstract}

Keywords: Parasitic Intestinal diseases, nutritional status, students, poverty, Peru.

\section{An Fac med. 2013;74(4):301-5}

\section{INTRODUCCIÓN}

La infección intestinal por parásitos es un serio problema de salud pública. La OMS estima que existen más de 2 billones de individuos infectados por parásitos a nivel mundial ${ }^{(1)}$. La presencia de esta infección no solo se asocia con niveles socioeconómicos bajos y condiciones sanitarias desfavorables (2), sino también a una alta prevalencia de ane- mia ferropénica, desnutrición y diarrea crónica ${ }^{(1)}$.

Estudios transversales realizados en niños en edad escolar del Perú han encontrado una prevalencia de infección por parásitos que varía entre 68 y $95 \%^{(3,4)}$, dependiendo de la localidad. El mayor porcentaje fue hallado en una localidad de Alto Marañón, zona de transición entre selva alta y selva baja. Sin embargo, no existen comunicacio- nes respecto a la prevalencia de infección por estos agentes en diversas zonas al interior de nuestro país.

La Encuesta Demográfica y de Salud Familiar halló que la prevalencia de desnutrición en la población preescolar era más alta en la selva ${ }^{(5)}$. Así se observa que en Huánuco, departamento caracterizado por poseer ecorregiones quechua y selva baja, existía 5,3\% de niños menores de 5 años con desnu- 
trición. Además, las enfermedades infecciosas parasitarias eran la segunda causa de morbilidad en niños en edad escolar ${ }^{(6)}$.

Otro factor que compromete el estado de salud del escolar de Huánuco es la pobreza. Según los Indicadores Básicos de Salud del Perú del año 2007, los valores de pobreza y pobreza extrema en Huánuco alcanzaron a $74,6 \%$ y $48,6 \%$ de la población, respectivamente ${ }^{(7)}$.

Por ello, el objetivo del presente trabajo fue determinar la asociación entre el nivel de pobreza y estado nutricional con la presencia de parásitos intestinales en los estudiantes de la institución educativa № 33164 Felipe Huamán Poma de Ayala, del caserío de Venenillo, Huánuco, Perú.

\section{MÉTODOS}

El diseño de estudio fue transversal, realizado entre los meses de octubre y diciembre de 2010.

El caserío Venenillo pertenece a la jurisdicción del centro poblado de Castillo Grande, distrito de Rupa Rupa, provincia de Leoncio Prado, departamento de Huánuco. Se encuentra a 649 metros de altura, de clima cálido, con temperatura media anual de $24^{\circ} \mathrm{C}$ y humedad relativa media de $85 \%$.

La población escolar estuvo constituida por 138 estudiantes inscritos en el único centro educativo de la comunidad. El tamaño de la muestra se obtuvo en base a una frecuencia estimada de infección por enteroparásitos de $95 \%$, con nivel de confianza de 0,95 y error máximo aceptable de 0,05, obteniéndose un tamaño de 46 estudiantes. Los sujetos de estudio fueron seleccionados mediante muestreo aleatorio simple a partir de la generación de números aleatorios de una lista general de alumnos. Se excluyó aquellos estudiantes que hubiesen recibido tratamiento antiparasitario o infusiones de plantas con este efecto, durante el mes previo a la toma de muestras ${ }^{(8)}$; del mismo modo, aquellos estudiantes que no entregaron las tres muestras o los cuestionarios incompletos.

Las muestras de heces fueron recolectadas durante tres días consecutivos, conservadas en formol al 10\% y transportadas a los laboratorios del Instituto de Medicina Tropical Daniel Alcides Carrión de la Universidad Nacional Mayor de San Marcos, donde fueron evaluadas mediante examen directo con lugol y técnica de sedimentación rápida de Lumbreras. No se empleó otros métodos, como el método de Graham o Kato-Katz, para la detección de especies específicas de parásitos ${ }^{(9)}$. Se excluyó las muestras contaminadas con agua u orina. La parasitosis fue expresada como número de parásitos de distinta especie encontrado por individuo.

Los estudiantes fueron tallados $\mathrm{y}$ pesados luego de entregar la última muestra, siguiendo los procedimientos consensuados.

El grado de pobreza fue establecido mediante el índice de necesidades básicas insatisfechas (NBI), el cual es utilizado por el Instituto Nacional de Estadística e Informática para establecer mapas de pobreza en el Perú. Las necesidades básicas se refirieron a características de la vivienda, hacinamiento, tipo de servicio higiénico, asistencia escolar y dependencia económica. Esta información fue recabada por medio de una ficha de recolección de datos. Aquellos individuos que no poseían necesidades básicas fueron clasificados como no pobres; quienes poseían una necesidad, pobres; y quienes poseían dos o más, pobres extremos ${ }^{(10)}$.

El grado de desnutrición fue determinado mediante el índice de Waterlow (relación talla/edad), según el cual la talla de los individuos fue comparada con la establecida para la edad según las curvas de la OMS. Estas últimas son aplicables en cualquier región del mundo. Los participantes fueron catalogados como sin desnutrición, o con desnutrición leve, moderada y severa ${ }^{(11)}$.

Posterior al análisis de las muestras, se entregó los resultados y medicación antiparasitaria gratuita en los casos requeridos.

Se calculó frecuencia y porcentaje para variables categóricas. La asociación de las variables ordinales fue determinada mediante el coeficiente gamma de Goodman y Kruskal. Se empleó el software SPSS 15 y se consideró como nivel de significancia 0,05.

La encuesta fue aprobada en el marco de la asignatura de Parasitología Médica Básica, del Departamento de Microbiología de la Facultad de Medicina San Fernando. El protocolo y los objetivos de la investigación fueron explicados a los padres y estudiantes; se obtuvo el consentimiento por escrito de los padres y el asentimiento verbal de los estudiantes. Asimismo, se obtuvo la aprobación de las autoridades de la institución educativa y el caserío.

\section{RESULTADOS}

La muestra calculada fue de 46 estudiantes, de los cuales 42 cumplieron los criterios establecidos. La edad promedio fue 10,8 \pm 4,2 años, y no se encontró diferencias entre género masculino $(11,3 \pm 4,2)$ y femenino $(10,2 \pm$ $4,3)(\mathrm{p}=0,37)$. Las características de la muestra son detalladas en la tabla 1.

Cuarenta y un individuos $(97,6 \%)$ estuvieron infectados con al menos una clase de parásitos. De ellos, 33 estudiantes $(78,6 \%)$ presentaron algún parásito patógeno y $37(88,1 \%)$, alguno no patógeno. Mientras que 29 estudiantes (69\%) tuvieron ambos tipos de parásitos.

Treinta y siete casos $(88,1 \%)$ presentaron poliparasitismo. El rango de infección múltiple fue entre dos y ocho parásitos de distinta especie por individuo, siendo la presentación más frecuente $(26,8 \%)$ la infección por cinco.

Los parásitos no patógenos y patógenos más frecuentes fueron Blastocystis hominis (83,3\%) y Ascaris lumbricoides $(42,9 \%)$, respectivamente. Las frecuencias según las especies de parásito se observan en la tabla 2. 
Tabla 1. Características de los estudiantes de la IE № 33164 , Huánuco-Perú. 2010.

\begin{tabular}{|lcc|}
\hline Característica & Frecuencia & Porcentaje \\
\hline Grupo de edad & & \\
\hline Preescolar (2-4 años) & 6 & 14,3 \\
\hline Escolar (5-11 años) & 10 & 23,8 \\
\hline Adolescentes (12-16 años) & 26 & 61,9 \\
\hline Género & & \\
\hline Masculino & 23 & 54,7 \\
\hline Femenino & 19 & 45,3 \\
\hline Nivel de pobreza & & \\
\hline No pobre & 4 & 9,5 \\
Pobre & 15 & 35,7 \\
Pobre extremo & 23 & 54,8 \\
\hline Grado de desnutrición & & \\
\hline No desnutrición & 2 & 4,8 \\
\hline Desnutrición leve & 0 & 0 \\
Desnutrición moderada & 4 & 9,5 \\
Desnutrición severa & 36 & 85,7 \\
\hline
\end{tabular}

Se encontró asociación significativa fuerte entre el nivel de pobreza y nivel de parasitosis $(\mathrm{p}=0,02$; gamma $=0,82)$. Sin embargo, no se evidenció asociación significativa entre el nivel de desnutrición y nivel de parasitosis $(\mathrm{p}=0,77)$.

\section{DISCUSIÓN}

La pobreza en la provincia de Leoncio Prado, a la cual pertenece Venenillo, ha sido considerada en $44,5 \%$, de acuerdo a los resultados obtenidos por el INEI ${ }^{(12)}$. Nuestro estudio determinó que $90,5 \%$ de la población de Venenillo vivía en condiciones de pobreza o pobreza extrema. Dicho porcentaje refleja que en la población de estudio existe vulnerabilidad en salud y desarrollo. Además, se encontró que la falta de servicios sanitarios fue la necesidad insatisfecha más frecuente, lo cual repercute en la predisposición a infecciones parasitarias ${ }^{(13)}$.

El 95,2\% de la población presentó valores bajos de talla para la edad, los que están relacionados a desnutrición crónica ${ }^{(14)}$, y se les considera un indi- cador de desarrollo de un país ${ }^{(5)}$. Sus consecuencias son el aumento de la morbilidad y mortalidad, así como un nivel en educación bajo ${ }^{(15)}$.

Se ha encontrado hasta 8 especies distintas de parásitos en un solo individuo. Esta cifra supera a la hallada en una población en Puno, en donde hubo un máximo de seis parásitos por individuo $^{(16)}$.

Tabla 2. Infección por parásitos en estudiantes del IE № 33164, Huánuco-Perú. 2010.

\begin{tabular}{c|c|c|c}
\hline Tipo de parásito & Enteroparásito & Frecuencia & Porcentaje \\
\hline \multirow{7}{*}{ Patógeno } & Ascaris lumbricoides & 18 & 42,9 \\
& Complejo E. histolytica / E. dispar & 11 & 26,2 \\
& Trichuris trichiura & 10 & 23,8 \\
& Giardia lamblia & 9 & 21,4 \\
& Uncinarias & 5 & 11,9 \\
& Hymenolepis nana $^{*}$ & 3 & 7,1 \\
& Enterobius vermicularis* $^{*}$ & 2 & 4,8 \\
& Strongyloides stercolaris* & 1 & 2,4 \\
\hline \multirow{4}{*}{ Comensal } & Blastocystis hominis & 35 & 83,3 \\
& Entamoeba coli & 30 & 71,3 \\
& Iodamoeba bütschlii & 22 & 52,4 \\
& Endolimax nana & 16 & 38,1 \\
\hline
\end{tabular}

*Determinado por examen directo con lugol y técnica de sedimentación rápida de Lumbreras.
El protozoario encontrado con mayor frecuencia fue B. hominis (83,3\%), hecho que coincide con estudios previos realizados en la población infantil de diversas comunidades nativas en la selva de Brasil ${ }^{(17-19)}$, las cuales comparten características geográficas y climáticas similares a Venenillo.

Además, se halló una elevada frecuencia de infección por parásitos comensales, siendo Entamoeba coli el de mayor frecuencia (71,3\%). Aunque dichos parásitos no causen repercusión sobre el estado de salud de los pacientes, reflejan la presencia de conductas de fecalismo. La alta frecuencia de dichas infecciones ha sido asociada a condiciones sanitarias deficientes ${ }^{(2,13)}$, como las de nuestra muestra.

Se evidenció que A. lumbricoides fue el helminto encontrado con mayor frecuencia $(42,9 \%)$, lo que se corrobora con estudios similares (20). Asimismo, refleja su alta prevalencia en la selva ${ }^{(21)}$. De ese modo, nuestro trabajo aporta datos actualizados a los pocos estudios realizados en dicha región.

El complejo Entamoeba histolytical dispar fue el protozoario patógeno encontrado más frecuentemente $(26,2 \%)$. Dicho hallazgo difiere de los resultados de un estudio realizado en Iquitos ${ }^{(22)}$, el cual encontró una mayor frecuencia de infección por Giardia lamblia. 
Dentro de los factores sociales, culturales y económicos que predisponen a la adquisición de parasitosis, la condición de pobreza repercute con mayor fuerza en países en vías de desarrollo como el Perú ${ }^{(23)}$. En diversos estudios realizados en países americanos ${ }^{(24-28)}$, se determinó correlación entre pobreza crítica y el porcentaje de individuos parasitados. En nuestra población, se evidenció asociación significativa y fuerte entre nivel de pobreza y parasitosis.

Del mismo modo, se ha determinado numerosos factores predisponentes a pobreza, entre los que destaca la falta de acceso a servicios sanitarios, la cual a su vez contribuye en los procesos continuos de infección y reinfección intestinal ${ }^{(26,27)}$.

En nuestro estudio se halló una frecuencia elevada de infección por parásitos y de desnutrición crónica en la muestra estudiada. La repercusión más importante sobre el estado de salud de los niños y adolescentes infectados es la limitación crónica en el desarrollo físico normal, dando lugar a diferentes grados de malnutrición, anemia por deficiencia de hierro, entre otras deficiencias nutricionales ${ }^{(29)}$. Dichas consecuencias varían dependiendo del tipo de parásito infectante. Así, las infecciones crónicas e intensas por A. lumbricoides generan importantes grados de desnutrición proteico-energética, además de distensión abdominal, anorexia y diarrea. En cambio, las infecciones crónicas por uncinarias ocasionan anemia por deficiencia de hierro, aunque infecciones intensas han sido relacionadas a cuadros de malabsorción intestinal por atrofia de vellosidades intestinales ${ }^{(29,30)}$.

Las infecciones intensas por Trichuris trichiura frecuentemente causan disentería crónica, aunque infecciones intensas y sostenidas han sido asociadas a disminución en el crecimiento y anemia de etiopatogenia no bien esclarecida ${ }^{(30)}$.

Pese a lo mencionado en los párrafos anteriores, el presente estudio no encontró diferencia significativa entre el número de parásitos y el grado de desnutrición. Resultado similar se obtuvo en otro estudio realizado en una comunidad con elevada presencia de parasitosis y desnutrición (31). Ello se podría explicar debido a que existen otros factores intervinientes sobre el estado nutricional, como el estado basal nutricional previo a la infección parasitaria, las infecciones concomitantes y el patrón de ingesta dietética durante la infección ${ }^{(23)}$. Asimismo, los factores socioeconómicos, culturales y sanitarios inherentes a la población estudiada tendrían una repercusión importante sobre el estado basal nutricional y por tanto sobre el impacto que la parasitosis genera en dicha población ${ }^{(32)}$.

Adicionalmente, se considera que la repercusión de los helmintos sobre el estado nutricional está en relación con la intensidad de infección, calculada por el conteo de huevos por gramo, índice que no fue evaluado en nuestro estudio ${ }^{(21,22)}$.

La frecuencia baja de infección por Enterobius vermicularis se ve afectada por la poca sensibilidad de los métodos empleados en nuestro estudio para su detección. Estos detectan entre 1 y $5 \%$ del total de casos positivos. La realización del examen de Graham brindaría una mayor precisión en la detección de este tipo de infección, lo cual se constituye como una limitación de nuestro estudio ${ }^{(33)}$.

En conclusión, se determinó una elevada frecuencia de pobreza, desnutrición e infección por parásitos en los estudiantes. Esta última presentó asociación significativa fuerte con el nivel de pobreza. Los resultados pueden ser importantes para brindar información acerca de las medidas de intervención que permitan disminuir las infecciones parasitarias, por medio del control de factores condicionantes presentes.

\section{REFERENCIAS BIBLIOGRÁFICAS}

1. World Health Organization. Deworming for Health and Development. Report of the Third Global Meeting of the Partners for Parasite Control. Geneva: World Health Organization; 2005.
2. Ngui R, Ishak S, Chuen C, Mahmud R, Lim Y. Prevalence and risk factors of intestinal parasitism in rural and remote West Malaysia. PLoS Negl Trop Dis. 2011;5(3):e974.

3. Marcos L, Maco V, Terashima A, Samalvides F, Gotuzzo E. Prevalencia de parasitosis intestinal en niños del valle del Mantaro, Jauja, Perú. Rev Med Hered. 2002;13(3):85-9.

4. Ibañez N, Jara C, Guerra A, Diaz E. Prevalencia de enteroparasitismo en escolares de comunidades nativas del Alto Marañón, Amazonas, Perú. Rev perú med exp salud publica. 2004;21(3):126-33.

5. Instituto Nacional de Estadistica e Informática. Informe principal Encuesta Demográfica y de Salud Familiar-ENDES Continua. 2010; Disponible en: http://proyectos.inei.gob.pe/endes/endes2010/ resultados/index.html.

6. Ministerio de Salud. Indicadores de morbilidad: Huánuco. Indicadores básicos en salud, 2007.

7. Ministerio de Salud. Indicadores socioeconómicos: Huánuco. Indicadores básicos en salud, 2007.

8. 8. Barahona L, Maguiña C, Náquira C, Terashima A, Tello R. Sintomatología y factores epidemiologicos asociados al parasitismo por Blastocystis hominis. Parasitol Latinoam. 2002;57(3-4):96-102.

9. Steinmann P, Du Z, Wang L, Wang X, Jiang J, Li $L$, et al. Extensive multiparasitism in a village of Yunnan Province, People's Republic of China, revealed by a suite of diagnostic methods. Am J Trop Med Hyg. 2008;78(5):760-9.

10. Cuba M. Necesidades básicas insatisfechas y uso de los servicios de atención primaria en Arequipa, Perú. MPA e-Journal Med Fam \& At Prim Int. 2008;2(2):85-90.

11. Guevara X. Estado nutricional de niños menores de 5 años de comunidades rurales y barrios urbanos del distrito de Chavín de Huántar. Paediatrica. 2003;5(1).

12. Instituto Nacional de Estadistica e Informática. Perú en cifras: Huánuco - Huánuco: Incidencia de la pobreza total según provincia. [Homepage] Lima: Instituto Nacional de Estadística e Informática; 2007 [citado 2012 July 10]; Disponible en: http:// www.inei.gob.pe/perucifrasHTM/inf-soc/cuadro.as $p$ ?cod=9068\&name $=d 10 \_16 \&$ ext $=$ jpg.

13. Navone G, Gamboa M, Oyhenart E, Orden A. Parasitosis intestinales en poblaciones MbyáGuarani de la Provincia de Misiones, Argentina: aspectos epidemiológicos y nutricionales. Cad Saúde Pública. 2006;22(5):1089-100.

14. Meneses A, Mendoza F. Características cefalométricas de niños con desnutrición crónica comparados con niños en estado nutricional normal de 8 a 12 años de edad. Rev Estomatol Herediana. 2007;17(2).

15. Martínez R, Fernández A. El costo del hambre: impacto social y económico de la desnutrición infantil en el Estado Plurinacional de Bolivia, Ecuador, Paraguay y Perú: Santiago de Chile. Naciones Unidas; 2009.

16. Marcos L, Maco V, Terashima A, Samalvides F, Miranda E, Gotuzzo E. Parasitosis intestinal en poblaciones urbana y rural en Sandia, departamento de Puno, Perú. Parasitol Latinoam. 2003;58(1-2):35-40.

17. Borges J, Alarcón R, Neto V, Gakiya E. Intestinal parasitosis in Indians of the Mapuera community (Oriximiná, State of Pará, Brazil): high prevalence of Blastocystis hominis and finding of Cryptosporidium sp and Cyclospora cayetanensis. Rev Soc Bras Med Trop. 2009;42(3):348-50. 
18. Albuquerque J, Queiroga A, Campos F, dosRamos S, Neves M, Sampaio E, et al. Intestinal protozoa and helminths among Terena Indians in the State of Mato Grosso do Sul: high prevalence of Blastocystis hominis. Rev Soc Bras Med Trop. 2007;40(6):631-4.

19. Nascimento S, Moitinho L. Blastocystis hominis and other intestinal parasites in a community of Pitanga City, Paraná State, Brazil. Rev Inst Med Trop Sao Paulo. 2005;47(4):213-7.

20. Supali T, Verweij J, Wiria A, Djuardi Y, Hamid F, Kaisar M, et al. Polyparasitism and its impact on the immune system. Int J Parasitol. 2010;40(10):1171-6.

21. Gyorkos T, Maheu M, Casapía M, Joseph S Creed $\mathrm{H}$. Stunting and helminth infection in early preschool-age children in a resource-poor community in the Amazon lowlands of Peru. Trans R Soc Trop Med Hyg. 2011;105(4):204-8.

22. Casapia M, Joseph S, Nuñez C, Rahme E, Gyorkos T. Parasite risk factors for stunting in grade $5 \mathrm{stu}$ dents in a community of extreme poverty in Peru. Int J Parasitol. 2006;36(7):741-7.

23. Cordero R, Infante B, Zabala M, Hage I. Efecto de las parasitosis intestinales sobre los parámetros antropométricos en niños de un área rural de
Rio Chico. Estado Miranda, Venezuela. RFM. 2009;32(2):132-8.

24. Borjas $P$, Arenas F, Angulo Y. Enteroparasitismo en niños y su relación con la pobreza y estado nutricional. CIMEL. 2009;14(1):49-54.

25. Rivero Z, Maldonado A, Bracho A, Gotera J, Atencio R, Leal M, et al. Enteroparasitosis en indigenas de la comunidad Japrería, estado Zulia, Venezuela. INCI. 2007:32(4):270-3.

26. Sultana S, Rahman M, Zaforullah M, Ali K. Intestina parasites and sanitary practices among the rura children. TAJ. 2007;20(1):1-5

27. Gamboa M, Navone G, Orden A, Torres M, Castro $L$, Oyhenart E. Socio-environmental conditions, intestinal parasitic infections and nutritional status in children from a suburban neighborhood of La Plata, Argentina. Acta Tropica. 2011;118(3):184-9.

28. Sangronis $M$, Rodriguez $A$, Pérez $M$, Oberto $L$, Navas P, Martinez D. Geohelmintiasis intestinal en preescolares y escolares de una población rural: realidad socio-sanitaria. Estado Falcón, Venezuela. Rev Soc Ven Microbiol. 2008;28(1):14-9.

29. Awasthi S, Bundy DA, Savioli L. Helminthic infections. BMJ. 2003;327(7412):431-3.

30. 30. Chiarpenello J. Infecciones por helmintos. Evid actual práct ambul. 2004;7(6):178-81.
31. Maia M, Fausto M, Vieira $E$, Benetton M, Carneiro M. Intestinal parasitic infection and associated risk factors, among children presenting at outpatient clinics in Manaus, Amazonas state, Brazil. Ann Trop Med Parasitol. 2009;103(7):583-91.

32. Quihui-Cota L, Valencia M, Crompton D, Phillips S, Hagan P, Diaz S, et al. Prevalence and intensity of intestinal parasitic infections in relation to nutritional status in Mexican schoolchildren. Trans R Soc Trop Med Hyg. 2004 98:653-9.

33. Requena I, Jiménez $Y$, Rodríguez N, Sandoval $M$ Alcala F, Blanco $\mathrm{Y}$ y col. Enterobius vermicularis en pre-escolares de un área suburbana en San Félix, estado Bolivar, Venezuela. Invest clin . 2007;48(3):277-86

Artículo recibido el 28 de abril de 2013 y aceptado para publicación el 4 de julio de 2013.

\section{Correspondencia:}

César Gabriel Berto Moreano

Correo electrónico: cesargabriel45@gmail.com 